\title{
Efeitos da Suplementação de Selênio nos Parâme- tros Glicêmicos da Síndrome do Ovário Policístico: Revisão Sistemática e Metanálise
}

\author{
Thayná Menezes Santos ${ }^{1}$, Keila Fernandes Dourado², Maria Izabel Siqueira de Andrade ${ }^{3}$
}

\begin{abstract}
RESUMO
Este trabalho tem como objetivo verificar, com base na literatura, os efeitos da suplementação de selênio nos parâmetros glicêmicos da síndrome do ovário policístico (SOP). O estudo é uma revisão sistemática e metanálise de ensaios clínicos randomizados com busca realizada por dois pesquisadores no período de janeiro a maio de 2019 , com delimitação temporal dos últimos dez anos, em base de dados Pubmed, Bireme e Medline, utilizando as palavras-chave síndrome do ovário policístico, selênio e resistência à insulina. Após a busca, os artigos foram filtrados de acordo com a temática proposta, utilizando o protocolo Prisma. Os critérios de inclusão foram: ensaios clínicos randomizados, realizados com mulheres com SOP, em uso de suplementação de selênio, incluindo os idiomas português, espanhol e inglês, sendo selecionados, ao final, três artigos para compor a revisão sistemática e metanálise. Foi aplicado índice Kappa interavaliadores. As análises estatísticas foram realizadas no software MedCalc com apresentação dos odds ratios e seus respectivos intervalos de confiança a $95 \%$. Os resultados mostram que a suplementação trouxe efeitos positivos em dois dos três artigos analisados, enquanto em um artigo o efeito foi negativo com aumento da resistência à insulina. Apesar disso, a análise da medida sumarizada pela metanálise demonstrou que não houve efeitos para o grupo de casos com a suplementação de selênio. Esta metanálise expôs que a suplementação de selênio não se configura como importante no controle glicêmico relacionado à SOP, posto que resultou em maior benefício para o grupo de controle dos estudos analisados.
\end{abstract}

Palavras-chave: Síndrome do ovário policístico. Selênio. Resistência à insulina.

EFFECTS OF SELENIUM SUPPLEMENTATION ON GLYCEMIC PARAMETERS OF POLYCYSTIC OVARY SYNDROME: SYSTEMATIC REVIEW AND META-ANALYSIS

\begin{abstract}
This study aimed to verify, based on the literature, the effects of selenium supplementation on the glycemic parameters of polycystic ovary syndrome (PCOS). The study is a systematic review and meta-analysis of randomized clinical trials with a search conducted by two researchers from January to May of 2019, with temporal delimitation of the last ten years, in a database such as Pubmed, Bireme and Medline, using the keywords polycystic ovary syndrome, selenium, and insulin resistance. After the search the articles were filtered according to the proposed theme, using the Prisma protocol. Inclusion criteria were: randomized clinical trials with women with PCOS using selenium supplementation, including the Portuguese, Spanish and English languages. Three articles were selected at the end to compose the systematic review and meta-analysis. We applied Kappa index interavers. Statistical analyzes were performed in MedCalc software with presentation of odds ratios and their respective $95 \%$ confidence intervals. The results indicate that supplementation had positive effects in two of the three articles analyzed, whereas in one article the effect was negative with increased insulin resistance. Despite this, the analysis of the measure summarized by the meta-analysis showed that there were no effects for the group of cases with selenium supplementation. This meta-analysis showed that selenium supplementation does not appear to be important in the glycoprotein control related to PCOS, since it resulted in greater benefit for the control group of the studies analyzed.
\end{abstract}

Keywords: Polycystic ovary syndrome. Selenium. Insulin resistance.

RECEBIDO EM: 31/10/2019

MODIFICAÇÕES SOLICITADAS EM: 29/3/2020

ACEITO EM: 30/3/2020

\footnotetext{
Nutricionista formada pela UFPE-CAV. Atua como residente no Programa de residência multiprofissional UFPE-CAV. http://lattes.cnpq.br/2402923633538000. https://orcid.org/0000-0002-2920-2373. thay.meenezes@gmail.com

Nutricionista formada pela UFPE. Professora-associada I do Núcleo de Nutrição do Centro Acadêmico de Vitória de Santo Antão (CAV) - UFPE na área de Nutrição Clínica. http://lattes.cnpq.br/2016583375679170. https://orcid.org/0000-0003-1346-8940. keiladourado@ig.com.br

3 Nutricionista formada pela UFPE. Professora-adjunta I da Universidade Federal de Alagoas-Ufal na área de Nutrição Clínica. http://lattes.cnpq.br/ 4699641000744646. https://orcid.org/0000-0003-1087-1320. andrademizabel@gmail.com
} 


\section{INTRODUÇÃO}

Segundo o Protocolo de Atenção Básica do Ministério da Saúde (BRASIL, 2016), nos sistemas de saúde tem-se priorizado o cuidado da mulher no campo da saúde reprodutiva, com foco na atenção ao pré-natal, parto, puerpério e planejamento reprodutivo. Também é prioridade de saúde pública a prevenção dos cânceres de colo do útero e de mama, patologias de grande relevância no cenário de saúde pública e bem consolidada na Atenção Básica. Apesar disso, o atendimento integral das mulheres com acolhimento de suas demandas e necessidades, garantia do acesso e respostas, ainda está em processo de consolidação.

Dentre as doenças mais comuns relacionadas ao sistema reprodutor feminino, destacam-se a candidíase, a síndrome do ovário policístico (SOP), endometriose, mioma uterino e os tipos de câncer previamente citados, cujas ações do Ministério da Saúde (BRASIL, 2012) têm sido cada vez mais específicas no intuito de diminuir sua prevalência. De modo especial, a SOP vem demonstrando um aumento de sua prevalência, trazendo grandes impactos na saúde da muIher. Concha et al. (2017), afirmaram que sua etiologia é multifatorial e se caracteriza como uma desordem genética complexa que atinge em torno de $6 \%$ a $18 \%$ das mulheres em idade reprodutiva, representando uma das principais causas de infertilidade nos dias atuais.

A SOP gera algumas complicações como desregulação do ciclo menstrual, câncer de endométrio e também alguns distúrbios metabólicos, como a disfunção da célula beta-pancreática e elevação de marcadores inflamatórios, como a Proteína $\mathrm{C}$ reativa (PCR). Mesmo com fisiopatologia não completamente conhecida, sabe-se que os fatores potenciais envolvem anormalidades na secreção do hormônio liberador de gonadotrofina e resistência à ação da insulina (SHOAEl et al., 2015).

Em razão do perfil inflamatório típico do quadro da SOP, estudos (WROBEL; POWER; TOBOREK, 2016; ALMONDES et al., 2010) têm associado a ação de micronutrientes antioxidantes e anti-inflamatórios na patologia, por exemplo: o selênio. Em 1957, Wrobel, Power e Toborek (2016) observaram que a suplementação em baixa dose de selênio impediu o processo de necrose em fígado de ratos e, a partir disso, pesquisas têm sugerido efeitos benéficos na manutenção da função imunológica e endócrina, ciclo metabólico e homeostase celular, apresentando, inclusive, ação anticarcinogênica. Em estudo de revisão, Almondes et al. (2010) concluíram que o selênio pode reduzir o risco de cân- cer, impedindo o ciclo tumoral, estimulando a apoptose e inibindo a migração e invasão celular tumoral, em suplementação com altas doses não tóxicas (400 $\mu \mathrm{g}$ ).

$O$ selênio é um mineral essencial encontrado no solo, água e alimentos e, embora seja um elemento que está naturalmente no organismo, seu nível endógeno oscila entre populações de diferentes áreas geográficas e em diversas idades numa mesma área, levando à questão de que fatores ambientais e internos teriam influência nos níveis séricos do mineral no corpo humano (WANG, 2014). Alimentos, como a Castanha do Pará, castanha de caju, carne bovina, por exemplo, são considerados alimentos-fonte (NAVARRO-ALARCON; CABRERA-VIQUE, 2008).

Mesmo sem o mecanismo de ação na SOP completamente esclarecido, ensaios clínicos (PIECZYNSKA; GRAJETA, 2015) mostram alterações em parâmetros glicêmicos, como índices HOMA-IR e HOMA-B, e diminuição da infertilidade feminina. Segundo a Federação Brasileira das Associações de Ginecologia e Obstetrícia (Febrasgo), micronutrientes, como o selênio, teriam efeitos no metabolismo da glicose, com ação na ativação da insulina ou apresentando efeito mimético, podendo ser associado com diminuição do risco cardiovascular em pacientes com síndrome metabólica, cuja prevalência gira em torno de $50 \%$ em mulheres com SOP (SOARES JÚNIOR; BARACAT; BARACAT, 2018).

É de suma importância o estudo ampliado sobre os efeitos da suplementação de selênio para fins terapêuticos, assim como de micronutrientes no geral, em especial relacionados ao quadro clínico da SOP, mostrando como a ciência da nutrição pode auxiliar na prevenção de doenças e na promoção à saúde da mulher.

Assim, o presente trabalho surge a partir da hipótese de que a suplementação de selênio em mulheres com SOP causa efeitos associados à melhoria do perfil glicêmico, sendo, consequentemente, importante na modulação do quadro clínico e no controle metabólico da doença, caracterizada por hiperglicemia e resistência à insulina e com o objetivo de verificar, com base na literatura, os efeitos da suplementação de selênio nos parâmetros glicêmicos da síndrome, assim como quais parâmetros são afetados pelo tratamento, dosagem, tempo de tratamento e forma de administração relacionados aos efeitos.

\section{METODOLOGIA}

Estudo de revisão sistemática da literatura conduzido nas bases de dados Medline (National Library of Medicine), Pubmed, Lilacs (Literatura Latino-ameri- 
cana e do Caribe em Ciências da Saúde), Scielo (Scientific Eletronic Library On-Line) e Bireme. As buscas foram realizadas no período de janeiro a maio de 2019. A pergunta condutora utilizada na pesquisa foi elaborada por meio da estratégia Picos, sendo apresentada da seguinte forma: "A suplementação de selênio é associada à melhora do perfil glicêmico e do quadro clínico de mulheres diagnosticadas com SOP?" A decisão de incluir a associação com modulação do perfil glicêmico e do quadro clínico da doença na revisão, deu-se porque essas direções são importantes para o entendimento aprofundado do tema, portanto a pesquisa incluiu trabalhos que abordam essas vertentes.

A busca teve como palavras-chave Polycystic ovary syndrome, selenium e insulin resistance, adaptando-se dependendo da base de dados, quando foram utilizadas buscas também combinando duas das três palavras-chave para ampliação da procura por artigos, e a estratégia de busca adotada para utilização nas bases de dados foi: [((polycystic ovary syndrome) AND selenium) AND insulin resistance].

Os critérios de inclusão dos estudos primários foram determinados previamente, sendo englobados ensaios clínicos randomizados, realizados com mulheres com SOP utilizando a suplementação de selênio, publicados nos idiomas português, espanhol ou inglês, com delimitação temporal de publicações entre os últimos dez anos.

Os critérios de exclusão foram determinados previamente, sendo excluídos artigos que não eram ensaios clínicos randomizados, estudos fora do recorte temporal, investigações experimentais e pesquisas que não analisaram o perfil glicêmico com a suplementação de selênio.

Foi usado o Protocolo Prisma (Preferred Reporting Items for Systematic reviews and Meta-Analyses), conforme exposto na Figura 1, para relatar a seleção dos estudos. Dois pesquisadores independentes realizaram as buscas e identificaram os potenciais estudos primários, sendo aplicado o índice Kappa ( $\mathrm{K}$ ) para verificação da concordância interavaliadores com relação à inclusão ou à não inclusão dos estudos na revisão sistemática. A extração dos dados foi feita no Microsoft Excel versão 2007 em um protocolo elaborado pelos pesquisadores para coleta de informações, como a dosagem, duração do tratamento e forma de administração do selênio, tamanho da amostra, quantificação de casos e controles com efeito após o tratamento, objetivos e limitações relatadas nos estudos analisados. Posteriormente, foi utilizado o checklist proposto por Downs and Black (DOWNS; BLACK, 1998) para avaliação da qualidade dos artigos selecionados para compor a presente revisão. Além disso, foi feita a revisão das referências dos artigos selecionados com o intuito de abranger mais estudos para a revisão.

Para a metanálise dos resultados utilizou-se o efeito fixo e random para obtenção da Forest Plot, a qual foi construída a partir do programa MedCalc statistical software versão 19.0.3. Foram apresentados os Odds Ratios (OR) e seus respectivos intervalos de confiança a 95\% (IC95\%), da associação entre a suplementação de selênio e a melhoria dos parâmetros glicêmicos na SOP, quando se adotou o nível de significância de $5 \%$ para verificação de significância estatística $(p<0,05)$. Em adição, a heterogeneidade dos estudos foi analisada por meio da inconsistência $\left(I^{2}\right)$ relacionada à proporção de variação na estimativa do efeito, sendo considerada a presença de heterogeneidade quando 12 fosse maior que $75 \%$ (STERNE, 2011). Todo o protocolo de busca foi cadastrado e aceito pela Prospero (Registro Internacional Prospectivo de Revisões Sistemáticas), obtendo número de registro CRD42019124418.

Figura 1 - Fluxograma de identificação e seleção dos artigos - Protocolo Prisma

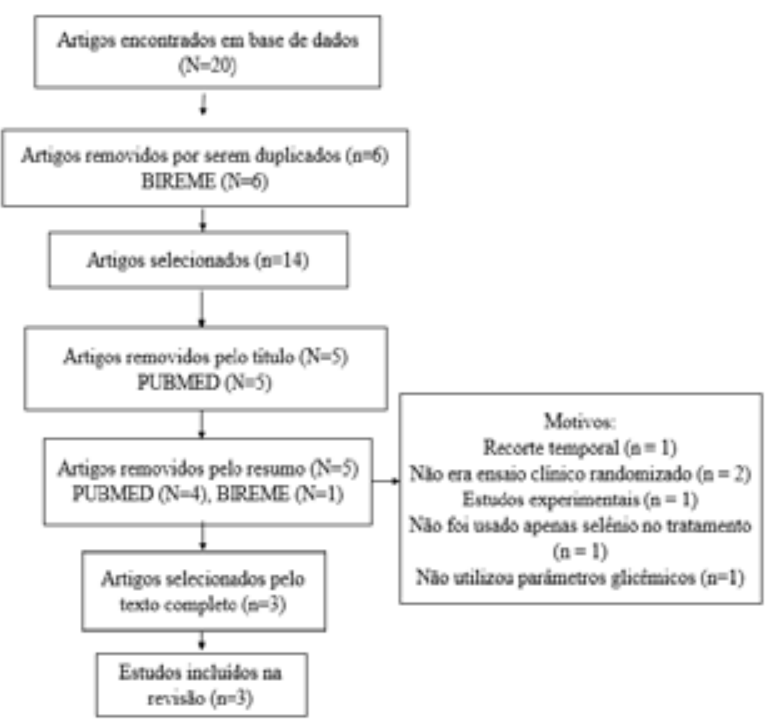

Fonte: Fluxograma criado por autoras desta revisão por meio de diretrizes do Protocolo Prisma.

\section{RESULTADOS}

Durante a busca, na Pubmed foram encontrados 13 estudos no total, quando 8 artigos foram incluídos após a avaliação dos títulos. Destes, 5 foram excluídos após leitura do resumo, após a constatação que 1 não abordava apenas a suplementação com selênio (dados não foram apresentados de forma isolada para o selênio), 1 não media parâmetros específicos de resistência à insulina, 2 estudos não caracteri- 
zavam ensaios clínicos randomizados e 1 não foi com humanos, sendo utilizados, portanto, 3 estudos para a análise dos resultados. Em outras bases de dados, como Scielo, não foram encontrados artigos que fossem incluídos no tema, enquanto na Bireme foram encontrados 7 estudos, dos quais 6 eram repetidos dos encontrados na Pubmed e 1 não contemplou o critério temporal para inclusão na revisão sistemática. A avaliação da concordância entre os dois pesquisadores para a seleção dos artigos originais em todas as etapas, evidenciou uma concordância perfeita $(k=1,0)$.

A partir da pontuação obtida pelo checklist Downs and Black, em que os artigos poderiam obter a pontuação máxima de 32, foi observado que os três artigos pontuaram 27 pontos, sendo considerada uma boa pontuação. Os artigos analisados perderam pontos nas questões relativas à validação externa, quando são analisados o tamanho da amostra e sua representatividade em relação à população total do local de estudo.

As características dos artigos incluídos nesta revisão estão apresentadas na Tabela 1 . Todas as investigações selecionadas analisaram se a suplementação de selênio estaria relacionada com a melhora de marcadores de resistência à insulina, típico de mulheres com SOP. De uma maneira geral, a amostra avaliada nos artigos foi composta por mulheres diagnosticadas com SOP em idade fértil, com faixa etária entre 18 e 40 anos, apresentando Índice de Massa Corporal (IMC) variando entre 25 e $28,39 \mathrm{~kg} / \mathrm{m}^{2}$. Todos os estudos incluídos nesta revisão apresentaram a mesma dosagem de suplementação de selênio, no valor de $200 \mu \mathrm{g} / \mathrm{dia}$, e dois dos três estudos incluídos trouxeram o mesmo tempo de tratamento, totalizando 8 semanas. Um estudo, particularmente, utilizou 12 semanas de tratamento e obteve um resultado negativo no índice HOMA-IR, parâmetro diagnóstico para a resistência à insulina.

De acordo com Modarres et al. (2017), a dosagem de selênio em $200 \mu \mathrm{g}$ diariamente proporcionou mudanças na expressão gênica de mulheres com SOP relacionadas ao perfil glicídico, com aumento do PPA-Y e diminuição da expressão de Glut-1 comparado ao grupo placebo. Jamilian et al. (2015), mostraram que a mesma dosagem de selênio provocou efeitos benéficos em relação à resistência à insulina com diminuição de HOMA-IR, HOMA- $\beta$ e diminuição adicional significativa dos níveis de triglicerídeos do grupo tratamento em comparação ao grupo placebo.

Tabela 1 - Características dos estudos incluídos na revisão e valor de escore da avaliação da qualidade dos artigos utilizados para a revisão sistemática e metanálise

\begin{tabular}{|c|c|c|c|c|c|c|c|c|}
\hline $\begin{array}{l}\text { Autor, ano/ } \\
\text { Local }\end{array}$ & $\mathrm{N}$ & $\begin{array}{c}\text { Forma de } \\
\text { suplementação }\end{array}$ & Dosagem & Tempo & Efeitos & Desfecho & Limitações & Escore \\
\hline $\begin{array}{l}\text { Mohammad } \\
\text { Hosseinzadeh } \\
\text { et al. (2015/ } \\
\text { Irã) }\end{array}$ & 60 & $\begin{array}{l}\text { Comprimido } \\
\text { enriquecido }\end{array}$ & $200 \mu \mathrm{g} / \mathrm{dia}$ & $\begin{array}{c}12 \\
\text { semanas }\end{array}$ & $\begin{array}{l}\text { Maior elevação } \\
\text { de HOMA-IR } \\
\text { e resistência } \\
\text { à insulina } \\
\text { no grupo } \\
\text { tratamento }\end{array}$ & $\begin{array}{l}12 \text { semanas } \\
\text { de tratamento } \\
\text { trouxe aumento } \\
\text { negativo sobre } \\
\text { resistência à } \\
\text { insulina }\end{array}$ & $\begin{array}{c}\text { Falta de } \\
\text { financiamento }\end{array}$ & 27 \\
\hline $\begin{array}{l}\text { Modarres et } \\
\text { al. (2017/Irã) }\end{array}$ & 40 & $\begin{array}{l}\text { Comprimido } \\
\text { enriquecido }\end{array}$ & $200 \mu \mathrm{g} / \mathrm{dia}$ & 8 semanas & $\begin{array}{l}\text { Aumento da } \\
\text { expressão } \\
\text { gênica de } \\
\text { PPA-Y, redução } \\
\text { de Glut-1 } \\
\text { no grupo } \\
\text { tratamento. }\end{array}$ & $\begin{array}{c}\text { Efeito benéfico } \\
\text { para expressão } \\
\text { gênica de } \\
\text { marcadores } \\
\text { como PPA-Y. }\end{array}$ & $\begin{array}{c}\text { Amostra } \\
\text { pequena. Falta } \\
\text { de análise do } \\
\text { nível sérico } \\
\text { de selênio } \\
\text { no início do } \\
\text { estudo }\end{array}$ & 27 \\
\hline $\begin{array}{l}\text { Jamilian et al. } \\
\text { (2015/Irã) }\end{array}$ & 70 & $\begin{array}{l}\text { Comprimido } \\
\text { enriquecido }\end{array}$ & $200 \mu \mathrm{g} / \mathrm{dia}$ & 8 semanas & $\begin{array}{c}\text { Diminuição } \\
\text { da insulina, } \\
\text { HOMA-IR, } \\
\text { HOMA- } \beta \text { e } \\
\text { diminuição } \\
\text { significativa de } \\
\text { triglicerídeos } \\
\text { no grupo } \\
\text { tratamento. }\end{array}$ & $\begin{array}{l}\text { Efeitos benéficos } \\
\text { sobre insulina, } \\
\text { HOMA-IR e } \\
\text { HOMA- } \beta \text { e perfil } \\
\text { lipídico. }\end{array}$ & Não informado & $\begin{array}{c}27 \\
\text { pontos }\end{array}$ \\
\hline
\end{tabular}

Escore: Pontuação da qualidade dos artigos, segundo recomendações propostas por Downs and Black.

Fonte: Tabela criada por todas as autoras deste artigo com base na análise dos dados dos estudos incluídos nesta revisão (JAMILIAN et al. (2015); MODARRES et al. (2017); MOHAMMAD HOSSEINZADEH et al. (2016). 
Tabela 2 - Médias de idade, índice de massa corporal e variação do índice HOMA-IR após o tratamento nas mulheres com SOP avaliadas nos estudos incluídos na revisão sistemática e metanálise

\begin{tabular}{l|lll} 
Autor, ano & $\begin{array}{l}\text { Média de idade } \\
\text { (tratamento vs. placebo) }\end{array}$ & $\begin{array}{l}\text { Média de IMC } \\
\text { (tratamento vs. placebo) }\end{array}$ & $\begin{array}{l}\text { Variação do HOMA-IR } \\
\text { (tratamento vs. placebo) }\end{array}$ \\
\hline $\begin{array}{l}\text { Mohammad Hosseinzadeh et } \\
\text { al. (2015) }\end{array}$ & $29.23 \pm 0.96$ vs. $28.90 \pm 1.17$ & $27.4 \pm 0.88$ vs. $28.39 \pm 0.72$ & $2.05 \pm 0.39$ vs. $-1.81 \pm 0.25^{*}$ \\
Modarres et al. (2017) & $31.1 \pm 4.7$ vs. $31.4 \pm 3.6$ & $26.5 \pm 4.1$ vs. $27.3 \pm 2.6$ & $-1.07 \pm 0.20$ vs. $+0.87 \pm 0.18^{*}$ \\
Jamilian et al. (2015) & $25.4 \pm 5.1$ vs. $25.7 \pm 4.8$ & $25.0 \pm 3.7$ vs. $25.2 \pm 4.1$ & $-1.15 \pm 1.81$ vs. $+0.42 \pm 3.09^{*}$ \\
\hline
\end{tabular}

$\mathrm{IMC}=$ Índice de massa corporal; HOMA-IR= Homeostatic Model Assessment for Insulin Resistance.

*p-valor<0,05; p-valor $<0,01$.

Fonte: Tabela criada por autoras deste artigo com base na análise dos dados dos estudos incluídos nesta revisão (JAMILIAN et al. (2015); MODARRES et al. (2017); MOHAMMAD HOSSEINZADEH et al. (2016).

Mohammad Hosseinzadeh et al. (2016) trouxeram um ensaio clínico em que o selênio possivelmente teria efeito negativo sobre marcadores de glicose e resistência à insulina, sendo evidenciado aumento do índice de HOMA-IR no grupo tratamento em comparação ao grupo placebo. Os autores não observaram mudanças significativas em relação a outros parâmetros analisados no estudo. A variação no índice HOMA-IR foi estatisticamente significante $(p<0,05)$ para todos os estudos avaliados, considerando a comparação da suplementação de selênio e placebo entre os grupos de casos e controles (Tabela 2).

A metanálise identificada pela forest plot está apresentada na Figura 2. Os resultados mostraram que dois dos três estudos incluídos apresentaram ausência de efeitos para o grupo dos casos, e a análise global demonstrou que a suplementação de selênio não confirmou melhorias nos parâmetros glicêmicos de mulheres com SOP, sendo encontrados maiores resultados no grupo controle em comparação aos casos (OR=2,96 [IC95\%:1,67;5,24]; $p<0,001$ ). Os resultados da metanálise demonstraram uma inconsistência $\left(I^{2}\right)$ de $95,5 \%$ e Pheterogeneidade $<0,0001$.

Figura 2 - Forest Plot da associação entre a suplementação de selênio vs. placebo e parâmetros glicêmicos em mulheres com SOP

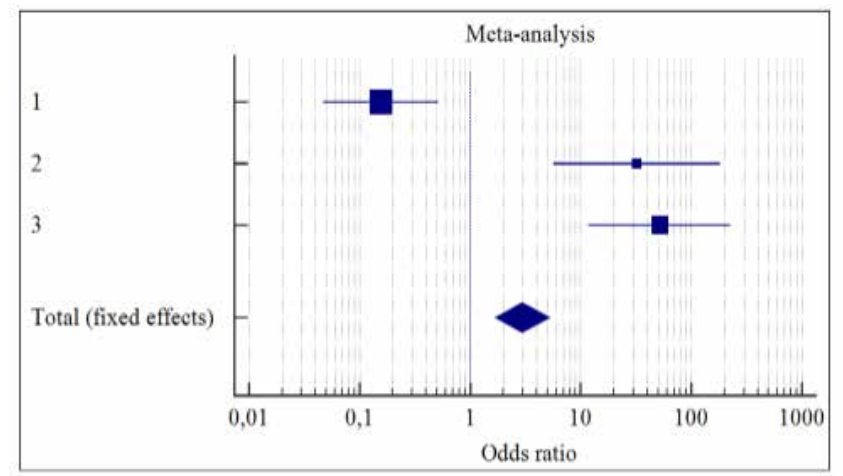

Fonte: Gráfico criado por meio da análise dos dados dos estudos incluídos nesta revisão ((JAMILIAN et al. (2015); MODARRES et al. (2017); MOHAMMAD HOSSEINZADEH et al. (2016)).

\section{DISCUSSÃO}

Mulheres com SOP possuem maior susceptibilidade a desenvolver resistência à insulina e diabetes mellitus, independente de obesidade (TEEDE et al., 2018). Além de anormalidades glicêmicas é comum o aparecimento de sintomas como hirsutismo, acne e anormalidades metabólicas, como o aumento de marcadores inflamatórios, hipertensão e alterações no perfil lipídico, como aumento de lipoproteínas (VLDL, $\mathrm{LDL}$ ) e triglicerídeos, o que, consequentemente, gera uma maior tendência a desenvolver doenças cardiovasculares (BARGIOTA; DIAMANTI-KANDARAKIS, 2012).

Apesar da ausência de efeitos nesta metanálise, alguns estudos mostraram que o selênio pode atuar como insulino-mimético por seu papel na regulação de enzimas da cascata de sinalização da insulina, na expressão de enzimas lipogênicas e no metabolismo de carboidratos no fígado (CHEN et al., 2015; MAO; TENG, 2013). Um estudo de revisão feito em 2018, por Fontenelle (2018), sobre o papel do selênio na resistência à insulina, mostrou que a ação do mineral na patogênese não está completamente elucidado, porém acredita-se que os principais mecanismos envolvem a regulação de concentrações intracelulares de espécies reativas de oxigênio (radicais livres) no metabolismo lipídico e glicídico. Além disso, mudanças nas concentrações de selênio contribuem significativamente no desenvolvimento da resistência à insulina (FONTENELLE, 2018). Pesquisas anteriores (BAHMANI et al., 2016; FARROKHIAN et al., 2016) elucidaram que a suplementação dietética com selênio pode reduzir concentrações séricas de insulina e o índice de resistência à insulina, indicado pelo HOMA-IR.

Um estudo feito por Mohammad Hosseinzadeh et al. (2016) trouxe um ensaio clínico em que o selênio possivelmente teria efeito negativo sobre os marcadores de glicose e de resistência à insulina, em razão do 
aumento do índice de HOMA-IR maior no grupo tratamento em comparação ao grupo placebo, e os autores não observaram mudanças significativas em relação a outros parâmetros observados no estudo. Um viés, contudo, pode ser considerado para este resultado, como a ausência das dosagens do selênio sérico. Além disso, pode-se observar que o tratamento foi feito por 12 semanas, diferentemente dos estudos que relataram efeitos positivos no grupo tratamento, podendo, assim, o tratamento com selênio ter efeito semelhante ao tóxico, exacerbando os níveis glicêmicos na SOP.

Um estudo feito em 2014 mostra efeitos negativos relacionados à intolerância à glicose e resistência à insulina quando o mineral é administrado em altas dosagens, superiores aos limites estabelecidos pelas diretrizes internacionais, abrindo a discussão para o cuidado com a administração do selênio em mulheres com SOP em busca de segurança terapêutica e maior evidência científica para validação do tratamento (WANG et al., 2014). Segundo Rayman (2012), quando há ingestão suficiente, gerando concentração sérica em torno de $80-120 \mu \mathrm{g} / \mathrm{L}$, o selênio atua como antioxidante e insulino-mimético, favorecendo a síntese e ação da insulina. Quando, porém, há concentração sérica superior a $120 \mu \mathrm{g} / \mathrm{L}$, o estado redox das células torna-se desequilibrado, podendo comprometer interações químicas envolvidas na cascata de sinalização da insulina (RAYMAN, 2012).

Como já visto nesta revisão, o controle dos níveis séricos de selênio é importante para evitar efeito tóxico por sua janela terapêutica ser pequena, comparada a outros minerais (FARROKHIAN et al., 2016). A toxicidade do selênio é dependente de diversos fatores, entre eles do composto e do método de administração utilizados, do tempo de exposição, do estado fisiológico, da idade do indivíduo e da interação com outros compostos (WANG et al., 2014).

Outros parâmetros podem ser considerados para análise de possíveis efeitos negativos e posterior controvérsia sobre o efeito do selênio na SOP, como os níveis séricos de selênio nas participantes dos estudos. Segundos os autores (JAMILIAN et al., 2015; MOHAMMAD HOSSEINZADEH et al., 2016), os estudos foram conduzidos em locais próximos (região de Teerã, no Irã), logo, as mulheres apresentavam características semelhantes, posto que eram de um mesmo país. Contraditoriamente, sabe-se que, mesmo dentro do mesmo território, é possível perceber uma grande diversidade biológica principalmente levando em conta o estado de globalização e influência de várias culturas. Assim, apesar de permitir um ajuste metodológico, nenhum local pode ser considerado igual em relação a seus habitantes. Este fato leva ao questionamento sobre as características biológicas das mulheres analisadas e sobre como o organismo respondeu ao tratamento de forma particular. De acordo com as recomendações internacionais de ingestão de selênio - a RDA (Recommended Dietary Allowance - Ingestão Diária Recomendada) -, mulheres com SOP possuem uma baixa ingestão do mineral em sua alimentação regular quando comparadas às mulheres saudáveis na mesma região (MOHAMMAD HOSSEINZADEH et al., 2016). Infere-se, dessa forma, que a baixa ingestão prévia dos alimentos ricos em selênio pode ser um dos motivos para que o tratamento não tenha alcançado o resultado esperado, pois as dosagens suplementadas atuariam apenas compensando as reservas nutricionais aquém do necessário, e não proporcionando, inicialmente, respostas metabólicas.

Em relação aos efeitos na resistência à insulina, os estudos apontam resultados discutíveis, posto que não é unanimidade em relação ao efeito benéfico do selênio. É importante pontuar a dificuldade de se encontrar boa quantidade de estudos que abordem esse tema com ensaios clínicos randomizados em humanos. É preciso, portanto, que mais estudos clínicos sejam feitos, com amostras representativas para que se tenha maior segurança e evidência científica sobre o efeito positivo ou negativo do selênio em todos os parâmetros relacionados à síndrome, uma vez que até o ano de 2015 somente era conhecido um estudo que abordasse a temática do efeito do selênio na SOP em relação a parâmetros glicêmicos, como indicado por Jamilian et al. (2015).

Os autores supracitados mostraram que a ação do selênio na SOP se configurou por melhora da função insulínica e melhora significativa de níveis de triglicerídeos e VLDL em grupo submetido ao tratamento com suplementação de selênio, porém sem efeito para outros parâmetros metabólicos. Este achado é importante, considerando que o quadro de hiperinsulinemia, presente em mulheres com SOP, gera um maior acúmulo de gordura corporal, alterando assim, o perfil lipídico. Como consequência, há maior síntese de citocinas inflamatórias que favorecem a resistência à insulina e risco de doenças (FREITAS; CESCHINI; RAMALLO, 2014). Os mecanismos moleculares para o desenvolvimento de resistência à insulina relacionada à obesidade e/ou sobrepeso, vêm sendo pesquisados intensamente; assim, evidências científicas relatam que o excesso de tecido adiposo e o consumo elevado de gorduras são capazes de sintetizar e ativar proteínas com ações inflamatórias que influenciam na via 
intracelular da insulina, causando prejuízos na translocação do transportador de glicose (Glut 4) para a membrana plasmática (HOLLAND et al., 2011).

Um estudo feito em 2015 por Razavi et al. mostra que o tratamento com selênio durante 8 semanas, com dosagem de $200 \mu \mathrm{g} / \mathrm{dia}$, trouxe efeitos positivos na diminuição de sinais e sintomas da SOP no grupo tratamento quando comparado ao grupo placebo, havendo redução da acne $(46,9 \%$ do grupo tratamento comparado a $12,5 \%$ no grupo controle), hirsutismo, alopécia (40,6\% grupo tratamento e $9,4 \%$ grupo controle) e melhora de parâmetros relacionados à infertilidade medida por taxa de gravidez ( $18,8 \%$ grupo tratamento e $3 \%$ grupo controle). Não houve, entretanto, resultados significativos entre os grupos em parâmetros relacionados ao estresse oxidativo, medido pelo valor de óxido nítrico (NO).

A melhora da resistência insulínica, associada à redução de peso, mostra efeito positivo sobre a regulação do ciclo menstrual e sobre a fertilidade, podendo ter uma melhor resposta do que o emprego de fármacos. A redução da hiperinsulinemia pode auxiliar na elevação da proteína carreadora dos esteroides sexuais (SHBG) e na redução da hiperandrogenemia. Além disso, há consenso que a identificação e devido tratamento da resistência à insulina/intolerância à glicose constituem uma boa forma de prevenção de diabetes mellitus e risco cardiovascular (SOARES JÚNIOR; BARACAT; BARACAT, 2018).

A partir da interpretação da metanálise construída, foi possível mostrar que a suplementação de selênio não é essencial no controle de parâmetros glicêmicos na SOP em razão de os resultados dos estudos mostrarem benefício maior para o grupo controle. Existe, portanto, o questionamento se a suplementação é, de fato, necessária ou se é mais viável que mulheres com SOP tenham uma ingestão adequada de selênio por meio de alimentos-fonte, enriquecendo sua dieta com alimentação mais saudável, posto que mudanças no padrão alimentar contribuem não somente nos parâmetros glicêmicos, mas, sim, no quadro clínico como um todo, pois, segundo a Federação Brasileira de Ginecologia e Obstetrícia (SOARES JÚNIOR; BARACAT; BARACAT, 2018), cerca de metade das mulheres com a SOP desenvolvem síndrome metabólica, que é influenciada diretamente pelo padrão alimentar.

De acordo com o estudo feito para esta revisão sistemática, pôde-se perceber que ainda há controvérsias em relação aos efeitos da suplementação de selênio nos parâmetros relacionados à hiperglicemia na SOP. É consenso na literatura, entretanto, que em pacientes com diabetes mellitus tipo 1 a suplementação de selênio se torna benéfica, podendo estar associada ao tratamento de complicações relacionadas à hiperglicemia, comum na SOP. É preciso salientar que o papel do selênio na regulação da insulina depende da forma química, dosagem e tempo de exposição ao tratamento, mesmo que seu mecanismo ainda não esteja completamente estabelecido na literatura (FONTENELLE, 2018) sendo, portanto, preciso mais estudos que expliquem a relação entre selênio e resistência à insulina em humanos.

Algumas limitações devem ser consideradas na leitura desta revisão, como a heterogeneidade dos estudos de base ( $I^{2}$ acima de $\left.75 \%\right)$, dificultando a interpretação dos resultados, o número reduzido de estudos disponíveis, as amostras pequenas, a falta de dosagem de selênio sérico nas mulheres estudadas, a ausência da análise do padrão dietético e a variabilidade temporal das investigações, que também foram fatores que podem ter contribuído para a alta heterogeneidade e para a ausência de efeitos da suplementação de selênio nas mulheres com SOP.

Apesar das limitações, foi possível refletir sobre os efeitos do selênio nos parâmetros glicêmicos da SOP, podendo compreender melhor as visões dos diferentes autores e dos estudos feitos até o momento da pesquisa. Foi importante observar que os ensaios clínicos randomizados, incluídos para esta revisão e posterior metanálise, utilizaram a mesma dosagem no tratamento (200 microgramas por dia), sendo realizados na mesma região geográfica.

A estimulação de uma dieta equilibrada e adequada em alimentos-fonte de nutrientes antioxidantes, como o selênio, torna-se viável para mulheres com a síndrome do ovário policístico, visando à meIhora do quadro e até à prevenção de complicações relacionadas à hiperglicemia e resistência à insulina, posto que a suplementação de selênio por meio de comprimidos se torna delicada pela necessidade do controle de níveis séricos.

Mais estudos do tipo ensaios clínicos randomizados, com maiores amostras, e em outros locais, devem ser feitos para um maior consenso sobre os efeitos da suplementação de selênio em relação à melhora dos parâmetros glicêmicos e também sobre a análise dos possíveis efeitos colaterais e toxicidade em relação à dosagem nas mulheres com SOP. Além disso, é importante a investigação dos efeitos deste micronutriente em outros parâmetros relacionados à SOP, como as alterações lipídicas e marcadores de estresse oxidativo, para melhor elucidar os efeitos bioquímicos e gênicos na resistência à insulina da SOP. 


\section{CONCLUSÃO}

Esta metanálise mostrou que a suplementação de selênio não se configura como importante no controle glicêmico relacionado à SOP, posto que resultou em maior benefício para o grupo controle dos estudos analisados.

Apesar dos achados do presente estudo, sabe-se que o efeito do selênio nos parâmetros glicêmicos e de resistência à insulina ainda não está totalmente elucidado na literatura, sendo importante o surgimento de mais estudos que expliquem sua ação em humanos. Além disso, caso haja estudos que monitorem a concentração sérica do selênio, é possível que a associação entre melhora do perfil glicêmico seja positiva, uma vez que necessita de maior financiamento.

Salienta-se que o padrão dietético, com o maior consumo de alimentos ricos em nutrientes antioxidantes, deve ser estimulado para a modulação do estado inflamatório, bem como para a prevenção das complicações geradas pelo quadro de resistência à ação da insulina.

\section{REFERÊNCIAS}

ALMONDES, K. G. S. et al. O papel das selenoproteínas no câncer. Rev. Assoc. Med. Bras., São Paulo, v. 56, n. 4, p. 484488, 2010.

BAHMANI, F. et al. Effect of selenium supplementation on glycemic control and lipid profiles in patients with diabetic nephropathy. Biol. Trace Elem., Londres, v. 172, n. 2, p. 282289, 2016.

BARGIOTA, A.; DIAMANTI-KANDARAKIS, E. The effects of old, new and emerging medicines on metabolic aberrations in PCOS. Ther Adv. Endocrinol. Metab., Los Angeles, v. 3, n. 1, p. 27-47, 2012.

BRASIL. Ministério da Saúde. Protocolo Clínico e Diretrizes Terapêuticas. Síndrome de ovários policísticos e hirsutismo/ acne. Portaria SAS/MS no 1.321 , de 25 de novembro de 2016.

BRASIL. Ministério da Saúde. Diretrizes metodológicas: elaboração de revisão sistemática e metanálise de ensaios clínicos randomizados. Brasília: Editora do Ministério da Saúde, 2012. 92 p.: il. (Série A: Normas e Manuais Técnicos).

CHEN, H. et al. Regulation of hepatic carbohydrate metabolism by selenium during diabetes. Chem Biol Interact, Amsterdam, v. 1, n. 232, p. 1-6, 2015.

CONCHA, F. C. et al. Epigenética del síndrome de ovário poliquístico. Revista Medicina del Chile, Santiago, v. 14, n. 5, p. 907-915, 2017.

DOWNS, S. H.; BLACK, N. The feasibility of creating a checklist for the assessment of the methodological quality both of randomised and non-randomised studies of health care interventions. Journal of Epidemiololy Community Health, Londres, v. 52, n. 6, p. 377-84, 1998.
FARROKHIAN, A. et al. Selenium supplementation affects insulin resistance and serum hs-CRP in patients with type 2 diabetes and coronary heart disease. Hormonal Metabology Research, Stuttgart, v. 48, n. 4, p. 263-268, 2016.

FONTENELLE, L. C. et al. The role of selenium in insulin resistance. Brazilian Journal of Pharmatology Science, São Paulo, v. 54, n. 1, p. 1-39, 2018.

FREITAS, M. C.; CESCHINI, F. L.; RAMALLO, B. T. Resistência à insulina associado à obesidade: Efeitos anti-inflamatórios do exercício físico. Revista Brasileira de Ciência e Movimento, São Paulo, v. 22, n. 3, p. 139-147, 2014.

HOLLAND, W. L. et al. Lipid-induced insulin resistance mediated by the proinflamatory receptor TLR4 requires saturated fatty acid-induced ceramide biosynthesis in mice. The Journal of Clinical Investigation, New York, v. 121, n. 5, p. 1.858-1.870, 2011.

JAMILIAN, M. et al. Metabolic response to selenium supplementation in women with polycystic ovary syndrome: a randomized, double-blind, placebo-controlled trial. Clin Endocrinol (Oxf), n. 82(6), p. 885-91, Original Article, Jun. 2015. DOI: $10.1111 /$ cen.12699.

MAO, J.; TENG, W. The relationship between selenoprotein $P$ and glucose metabolism in experimental studies. Nutrients, Basel, v. 5, n. 6, p. 1.937-1.948, 2013.

MODARRES, Z. et al. The Effects of Selenium Supplementation on Gene Expression Related to Insulin and Lipid in Infertile Polycystic Ovary Syndrome Women Candidate for In Vitro Fertilization: a Randomized, Double-Blind, Placebo-Controlled Trial. Journal of Trace Elements in Medicine and Biology, n. 183(2), p. 218-225, Sep. 2017

MOHAMMAD HOSSEINZADEH, F. et al. Effects of selenium supplementation on glucose homeostasis and free androgen index in women with polycystic ovary syndrome: A randomized, double blinded, placebo controlled clinical trial. Journal of Trace Elements in Medicine and Biology, v. 34, p. 56-61, Mar. 2016.

NAVARRO-ALARCON, M.; CABRERA-VIQUE, C. Selenium in food and the human body: A review. Science Total Enviroment, Londres, v. 40, n. 3, p. 115-141, 2008.

PIECZYNSKA, J.; GRAJETA, $H$. The role of selenium in human conception and pregnancy. Journal Trace Elements Medicine and Biology, New York, v. 29, n. 3, p. 31-38, 2015.

RAYMAN, M. P. Selenium and human health. Lancet, Londres, v. 379, n. 9.822, p. 1.256-1.268, 2012.

RAZAVI, M. et al. Selenium Supplementation and the Effects on Reproductive Outcomes, Biomarkers of Inflammation, and Oxidative Stress in Women with Polycystic Ovary Syndrome. Horm Metab Res, Epub, 2015. DOI: 10.1055/s-00351559604.

SHOAEI, T. et al. Effects of Probiotic Supplementation on Pancreatic $\beta$-cell. Function and C-reactive Protein in Women with Polycystic Ovary Syndrome: A Randomized Double-blind Placebo-controlled Clinical Trial. International Journal of Preventive Medicine, Isfahan, v. 1, n. 4, 2015. 
SOARES JÚNIOR, J. M.; BARACAT, M. C. P.; BARACAT, E. C. Repercussões metabólicas: quais, como e porque investigar? In: FEDERAÇÃO BRASILEIRA DAS ASSOCIAÇÕES DE GINECOLOGIA E OBSTETRÍCIA (FEBRASGO). Síndrome dos ovários policísticos, São Paulo, n. 4, p. 29-39, 2018. (Série Orientações e Recomendações Febrasgo). ISBN 978-8594091-05-5.

STERNE, J. A. C. et al. Recommendations for examining and interpreting funnel plot asymmetry in meta-analyses of randomised controlled trials. BMJ, v. 3, n. 43, 2011.

TEEDE, H. et al. International PCOS Network. Recommendations from the international 39 evidence-based guideline for the assessment and management of polycystic ovary syndrome. Humam Reproduction, New York, v. 34, n. 9, p. 23-29, 2018.

WANG, N. et al. Supplementation of Micronutrient Selenium in Metabolic Diseases: Its Role as an Antioxidant. Hindawi Oxidative Medicine and Cellular Longevity. Londres, v. 7, n. 4, 58-65, 2017.

WANG, X. et al. High selenium impairs hepatic insulin sensitivity through opposite regulation of ROS. Toxicologicy Leter, New York, v. 2, n. 24, p. 16-23, 2014.

WROBEL, J. K.; POWER, R.; TOBOREK, M. "Biological activity of selenium: revisited," IUBMB Life, New York, v. 68, n. 2, p. 97-105, 2016. 